\title{
Planning of scooping position and approach path for loading operation by wheel loader
}

\author{
Shigeru Sarata, Yossewee Weeramhaeng and Takashi Tsubouchi
}

\begin{abstract}
Our group developed a method for planning of scooping point and approach path as a part of our ongoing research on autonomous loading operation of wheel loader at construction sites.

The planning of scooping position and direction is obtained through processing pile model. The pile model represents shape and volume of the pile, changes of shape and volume by scooping, and falling behavior accompanied scooping. Scooping direction should be perpendicular to slope of the pile to avoid unbalance of resistance force around the center line of the bucket. The resistance force imposed on the bucket is estimated using the pile model and bucket trajectory model. The scooping direction with least unbalance of resistance force is selected.

$\mathrm{V}$ shape path between the scooping position and the loading position to dump track is composed of straight lines and clothoid curves. For given scooping position and loading position, the path with the least length is produced by the proposed planning method using optimization by Lagrange multiplier.

For selection of the next scooping point, $\mathrm{V}$ shape paths for candidate of scooping points are planned by proposed method.
\end{abstract}

Index Terms-loading operation, path generation, scooping wheel loader

\section{INTRODUCTION}

The front end wheel loader ("loader" hereinafter) is one of the major loading machines for earth moving operation in construction, mining and other many fields. The worksites in construction and mining include irregularly shaped materials that change shape as operations progress, such as piles of sand, soil, and fragmented rock. The unmanned systems in these fields should be intelligent systems with the capacity to decide their actions based on the changing conditions at worksites.

Our group developed a method for planning of scooping point and approach path as a part of our ongoing research on autonomous loading operation of wheel loader at construction sites[1][2]. For an autonomous system, planning function is one of the essential elements. Several researches have been conducted on automatic operation system of wheel loader [3][4]. These developed systems employed a guidance method

S. Sarata is with the National Institute of Advanced Industrial Science and Technology (AIST), Tsukuba, Ibaraki 305-8564 Japan.

Y. Weeramhaeng is with The University of Tsukuba, Tsukuba, Ibaraki 305-8573 Japan

T. Tsubouchi is with The University of Tsukuba, Tsukuba, Ibaraki 305-8573 Japan or a teaching-playback method for traveling. Path generation function was not included.

The most common path between the scooping position and the loading position on the dump truck is "V Shape" path including switchback in the middle of the path as shown in Fig.1. The loading operation is carried out by repetitions of this cycle of scooping and loading. The scooping position of each cycle is changed as operation progress. The determination of the scooping direction and the generation of the path between the scooping point and the loading point are essential functions for an autonomous loading system. This paper describes a planning method of $\mathrm{V}$ shape path including determination of the scooping direction based on the shape of the pile.

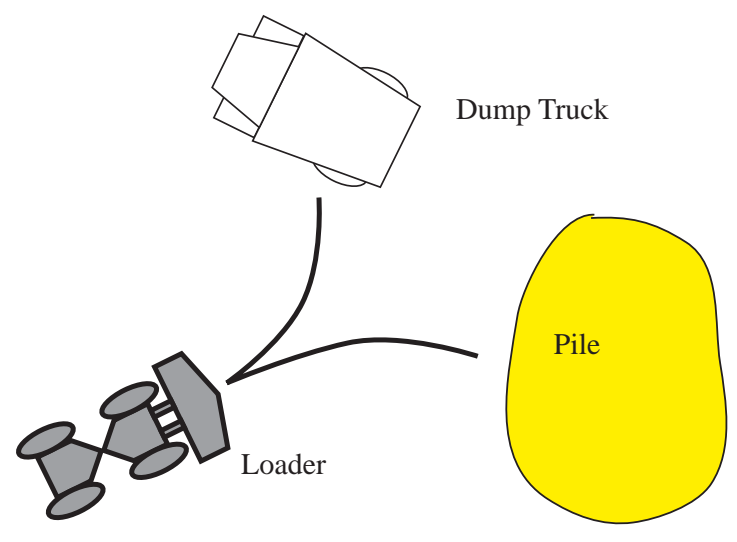

Fig.1 V shape Path

\section{Model of The Pile And The SCOOPING DiRECTION}

\section{A. Resistance Force Applied On The Bucket}

The bucket is subjected to large resistance force from the pile during scooping. The magnitude and direction of the resistance force are affected by the shape of the pile and trajectory of the bucket. The resistance force can be classified into several elements of force. The dominant elements are the penetrating force and the force required to move the material in front of the bucket. From the results of the basic experiments on the resistance force between the bucket and material in the pile, both of the forces have close relation to depth of the material of the pile at the tip of the bucket.

The depth of the material at the tip of the bucket changes as the bucket advances during the scooping operation. The bucket trajectory of a standard scoop is composed of the three sections shown in Fig. 2. In the first section, the base of the bucket 
remains flat against the ground. As the loader advances on its wheels, the bucket penetrates the pile from points A to B (section 1). From points B to $C$ (section 2), the bucket arm moves the tip of the bucket upward along a line or curve and increases the tilt. Once filled with the piled material, the bucket moves upward almost vertically from points $\mathrm{C}$ to $\mathrm{D}$ (section 3 ). The horizontal length of scooping, $\mathrm{AB}$ and $\mathrm{BC}$ ', depends on the capacity of the bucket and shape of the pile. In a standard scoop, the total length of scooping AC' is about 1.5 times as long as the length of the bucket base.

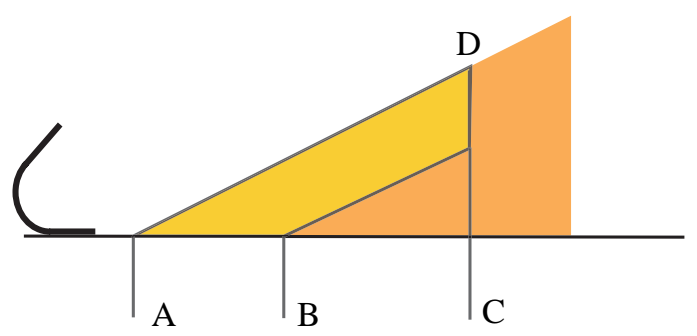

Fig.2 Bucket Motion trajectory

\section{B. Pile Model}

The column model [1] is a useful system for estimating the interaction between the bucket and piled material. Though simple in structure, the model can represent the shape of the pile, as well as changes in both shape and volume. Fig.3 shows the structure of the column model. The working area is tessellated into sections, each forming the base of a column. The height of each column represents the height of the pile at that position. The sizes of the sections and unit of height have no relation to the particle or fragment diameter of the material making up the pile.

\section{Fig.3 Column Model}

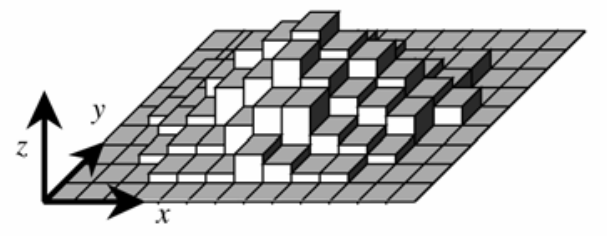

The column model is formed based on the result of threedimensional shape measuring of the pile. Stereo-vision system with two CCD cameras is used for the shape measuring. As the pile of gravel or fragmented rock has random texture on the surface, correlation method is employed for finding corresponding points on the two images taken by CCD cameras. $3 \mathrm{D}$ reconstruction is obtained through trigonometry with disparity.

\section{Determination of Scooping Direction}

If possible, the scooping direction should be perpendicular to slope of the pile (Fig. 4 (a)). If the scooping direction is far from perpendicular, as shown in (b), the resistance force around the center line of the bucket is asymmetrical, imposing undesirable stress on the bucket link mechanism.

The unbalance of the resistance force imposed on the bucket is estimated by estimating the resistance force at each point on tip of the bucket using the column model and bucket trajectory model (Fig. 6). The scooping direction with the least unbalance of resistance force should be selected to obtain the proper scooping motion.

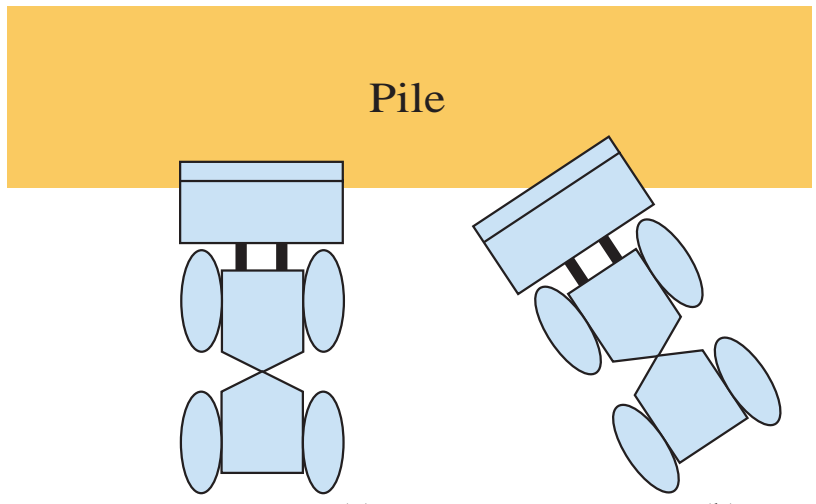

(a)

(b)

Fig.4 Scooping Angle

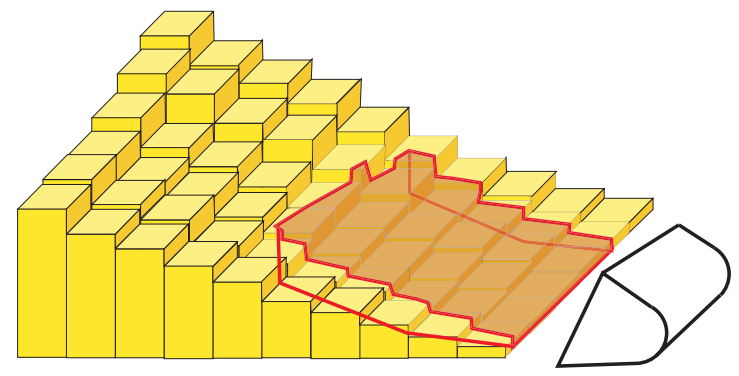

Fig.5 Column model and scooping area

Discreet points are plotted on the scooping area as shown in Fig. 6. Let $w_{j}$ be the distance from the center of the bucket and $h_{i j}$ be the average depth of the material piled in the unit width at that point. The variable $m_{i j}$ is defined as:

$m_{i j}=h_{i j} \cdot w_{j} \quad$.

Thus, $m_{i j}$ is a value related to the moment around the center of the bucket. $M$ is defined as the sum of all $m_{j i}$ values in scooping area.

$M=\sum m_{i j}$

$M$ is the sum of all values related to the moment applied on the bucket during one scooping cycle. $M$ changes with changes in the scooping direction from the same initial scooping position. The smallest $M$ is expected when the scooping is executed in a direction normal to the slope, but the value is also affected by the shape of the scooping area on the pile. The scooping direction with the smallest $M$ is determined by comparing the estimated $M$ values in different directions around the normal vector in the section neighboring the initial scoop position. 


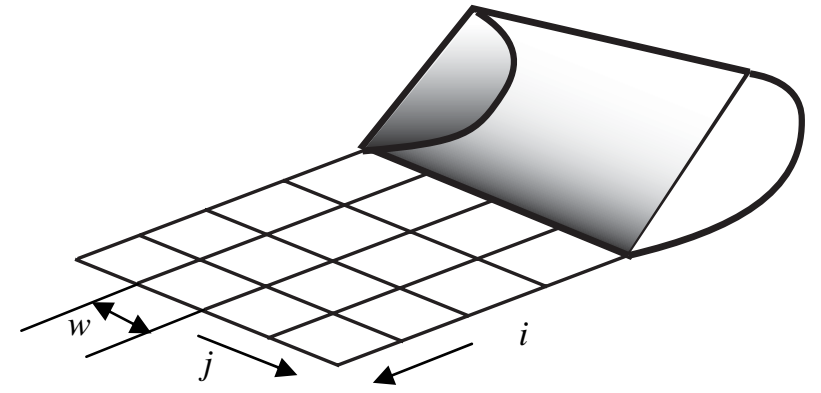

Fig. 7 Discreet points for estimation of resistance force

\section{V SHAPE PATH PLANNING}

\section{A. Steering System of The Wheel Loader}

The wheel loader employs an articulate steering system of the type shown in Fig. 7. The body of the loader is separated into a front part and rear part connected by a center pin. The angle around the center pin is controlled by hydraulic cylinders. The placement of the front and rear axles at equal distances from the center pin ensures that the front and rear wheels travel over the same path, conferring high mobility on off-road surfaces such as mud or soft soil.

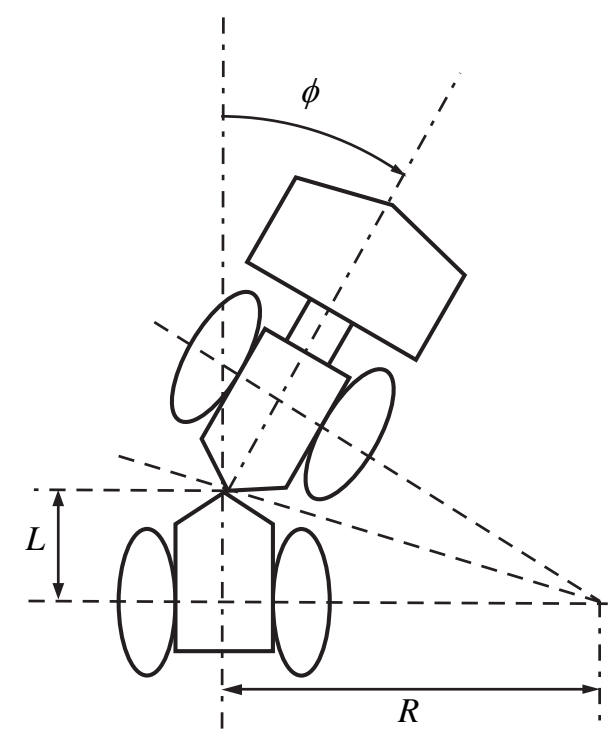

Fig. 7 Steering system

The relation between the articulate angle $\phi$ and curvature $\kappa$ is described by (1). Where $\mathrm{L}$ is the distance from the center pin to the front or rear axle.

$\phi=2 \tan ^{-1}(\kappa L / 2)$

When $\phi$ is small, curvature $\kappa$ is proportional to $\phi$ linearly. The operational range of the articulate angle is about 40 degrees. The relation between $\kappa$ and $\phi$ is almost linear in this range.

\section{B. Path Planning With Path Elements}

A path from the given initial position to a final position can be easily generated by combining path elements such as straight lines and curves. In the proposed method for path planning, we use a clothoid with a curvature proportional to the length of the path as a curve element. This clothoid curve can be described by the following formulae:

$$
\begin{aligned}
& x(s)=\int_{s_{0}}^{s} \cos \left(k s^{2}\right) d s \\
& y(s)=\int_{s_{0}}^{s} \sin \left(k s^{2}\right) d s,
\end{aligned}
$$

where $s$ is the length of the path and $k$ is a coefficient related to the sharpness of the path.

If the curvature changes as shown in Fig. 8(a), the path becomes the same clothoid joined in reverse directions, or what we call a "symmetrical clothoid" (Fig. (b)). Given that the curvature is zero at both ends of the symmetrical clothoid, the curve can be connected to straight lines with continuously.

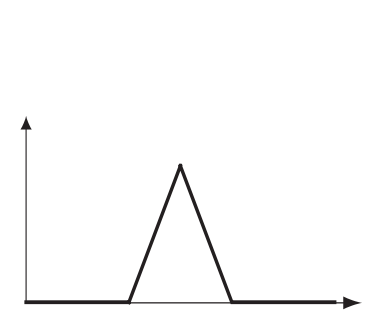

(a) curvature

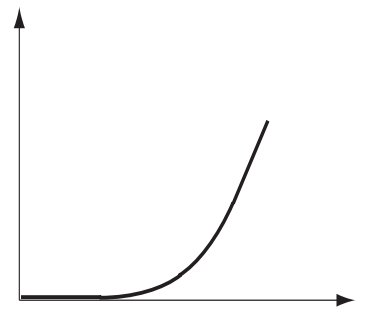

(b) Path
Fig. 8 Symmetrical clothoid

Here we consider a V shape path constituting two symmetrical clothoids and straight lines. For convenience, we assume that the loader moves from the scooping position to the loading position and the loader is initially positioned on the origin of the coordinate system and moves in a negative direction on the $\mathrm{x}$ axis, as shown in Fig. 9. The grey zone in Fig. 9 is the area that can be reached via the combination of straight line $l_{1}$, symmetrical clothoid $c_{1}$, straight line $l_{2}$, symmetrical clothoid $c_{2}$, and straight line $l_{3} . l_{1}, l_{2}$ and $l_{3}$ are the directions of the loader at the initial position, at the switchback, and at the final position, respectively. The straight line segments can take a negative direction for the backwards run of the loader. From requirement of the operation, the line segment $l_{1}$ should be negative and $l_{3}$ should be positive.

\section{Path Planning}

When the end position and direction of the symmetrical clothoid are given, the corresponding clothoid can be obtained by solving formulae (4) and (5). These formulae cannot be solved analytically, however, as both are Fresnel integrals. Numerical solutions can be reached using the Taylor extension, but the complexity of the whole procedure calls for simplification. Here we simplify the procedure by limiting the curvature $\kappa$ to several values. This provides a large practical advantage. 


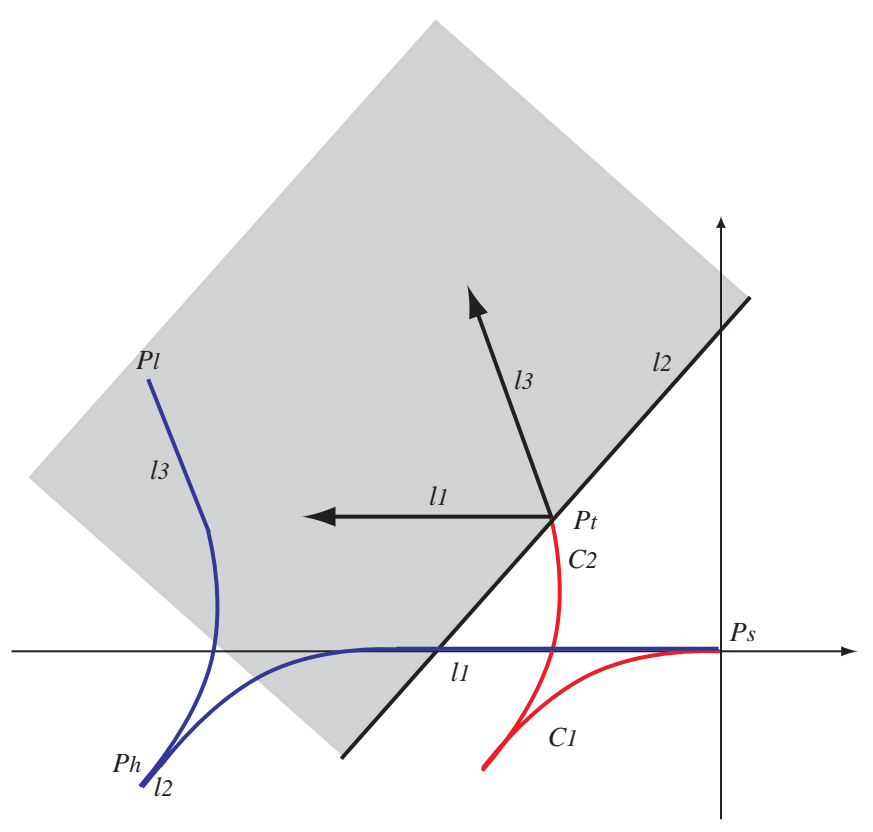

Fig. 9 Zone reachable via the combination of clothoids and straight lines

The path planning proceeds in the following steps. Let $P_{s}$ be the scooping position, $\theta_{s}$ be direction at that position, $P_{e}$ be the loading position, $\theta_{e}$ be direction at that position and $\theta_{h}$ be direction at $P_{h}$ :the switch back point or the end of $c_{1}$. $\theta_{h}$ takes value between $\theta_{s}$ and $\theta_{e}$. Path consists of $l_{1}-c_{1}-l_{2}-c_{2}-l_{3}$. In the planning procedure, the path is separated into $c_{1}-c_{2}$ and $l_{1}-l_{2}-l_{3}$. At the first step, set $\kappa$ at a certain value and calculate $c_{1}$ : the symmetrical clothoid which turn the loader by angle $\theta_{h}-\theta_{s}$. Next, calculate $c_{2}$ for angle $\theta_{e}-\theta_{h}$ in the same manner and connect $c_{1}$ and $c_{2}$ at the switch back. In the second step, generate the path $l_{1}-l_{2}-l_{3}$ between the end point of $c_{1}-c_{2}: P_{t}$ and the scooping point $P_{e}$ with optimization on length of $l_{1}-l_{2}-l_{3}$.

Function $Z$ for the optimization of the length $l_{1^{-}} l_{2^{-}} l_{3}$ is defined in (6),

$Z=l_{1}^{2}+l_{2}^{2}+l_{3}^{2}$

Constraint conditions are:

$C_{1}=\left(x_{e}-x_{t}\right)-\left(l_{1}+l_{2} \cos \theta_{t}+l_{3} \cos \theta_{e}\right)$

$C_{2}=\left(y_{e}-y_{t}\right)-\left(l_{2} \sin \theta_{t}+l_{3} \sin \theta_{e}\right)$.

Function $F$ on the aforesaid is defined as:

$F=Z+\mu_{1} C_{1}+\mu_{2} C_{2}$,

where $\mu_{1}$ and $\mu_{2}$ are Lagrange's multipliers. From these equations, the combination of $l_{1}-l_{2}-l_{3}$ with the shortest length is obtained.

Fig.10 shows an example of process of the path planning. The scooping point $P_{s}$ is located at $(00)$ and the angle $\theta_{s}=-10$. The loading point $P_{e}$ is located at (-1000 1000) and the angle $\theta_{e}=80$. The bold line represents the shortest path at $\theta_{h}=48$. The thin lines represent the path at $\theta_{h}=10$.and $\theta_{h}=70$.

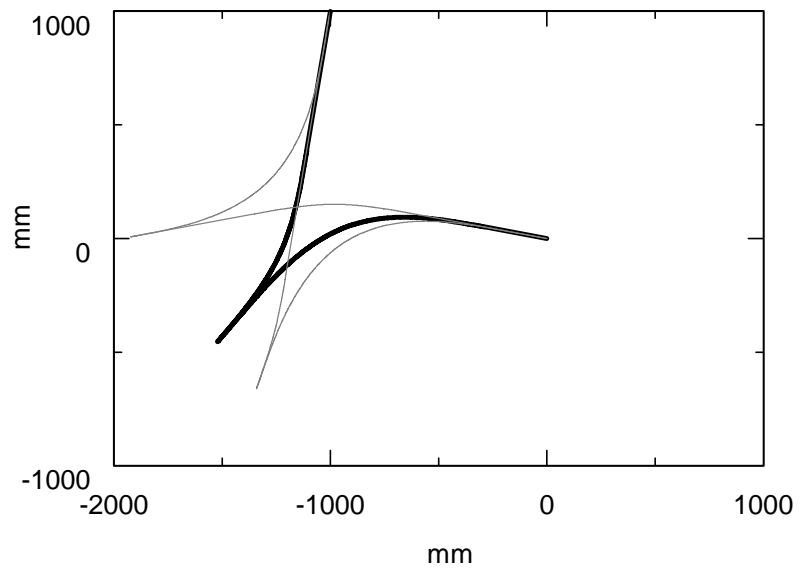

Fig.10 Process of path planning

The total length of the path at every 2 degree of $\theta_{h}$ is calculated out as shown in Fig.11. In the example case, the length of the path changes from $3973 \mathrm{~mm}$ at $\theta_{h}=-10$ to $3307 \mathrm{~mm}$ at $\theta_{h}=48$. The length of $l_{1}$ and $l_{3}$ are shown in Fig.12. Black triangles and gray squares represent length of $l_{1}$ and $l_{3}$ respectively. $l_{1}$ takes negative value in the whole range of $\theta_{h}$, but $l_{3}$ takes negative value at $\theta_{h}=-10 \sim 0$. These results do not fill the requirement for $\mathrm{V}$ shape path. The results at $\theta_{h}=0 \sim 80$ fill the requirement and the total length becomes the shortest at $\theta_{h}=48$.

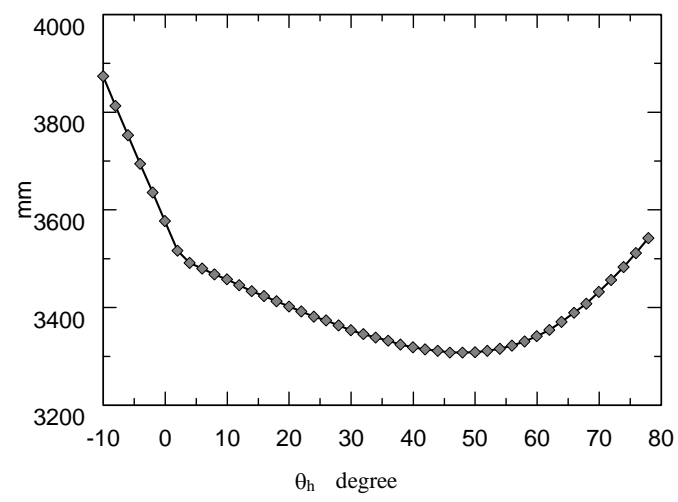

Fig.11 Total length of the path

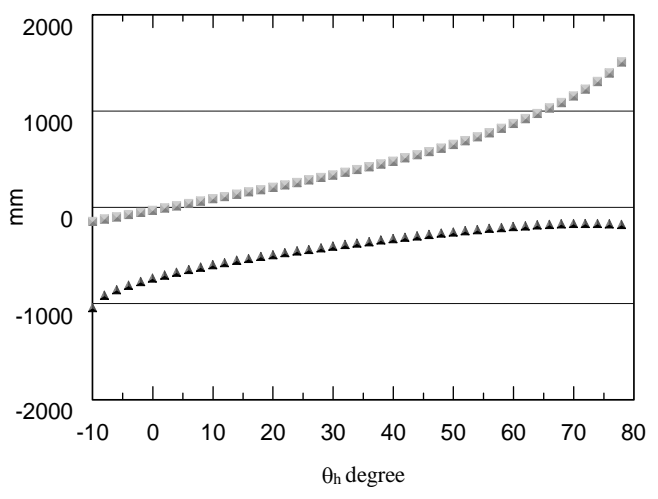

Fig.12 Length of $l_{1}$ and $l_{3}$ 


\section{V Shape PATH PLANNING WITH EXPERIMENTAL MODEL}

The proposed method is evaluated using the experimental model called YAMAZUMI-2 (YZ-2, Fig. 13) and a pile of fragmented granite. The YZ-2 has the same structure and function of a wheel loader. A distance of $270 \mathrm{~mm}$ separates the front and rear axles and the bucket measures $250 \mathrm{~mm}$ in width. The experimental pile consisted of $5 \mathrm{~mm}$ fragments piled over a surface area of about $1000 \mathrm{~mm}$ to a height of about $300 \mathrm{~mm}$. Two CCD cameras were attached to the YZ-2 to measure the shape of the pile by a stereo-vision system. In following planning, the steering angular velocity is set at 5 degree/L.

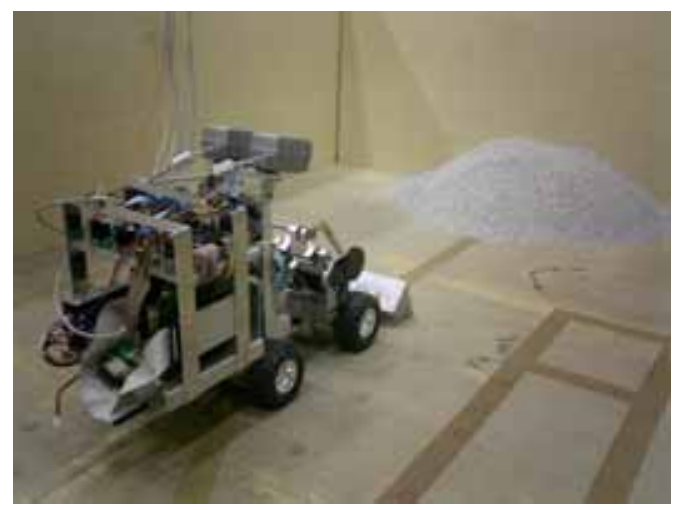

Fig. 13 Experimental model YZ-2

Fig. 14 shows the image of the pile by the CCD camera. The shape of the pile was obtained by applying the correlation method on the images. A column model of the experimental pile is formed as previously mentioned. The model consisted of $300 \times 300$ columns, each measuring $5 \times 5 \mathrm{~mm}$. The column model represented a $1500 \times 1500 \mathrm{~mm}$ area in the experimental field.

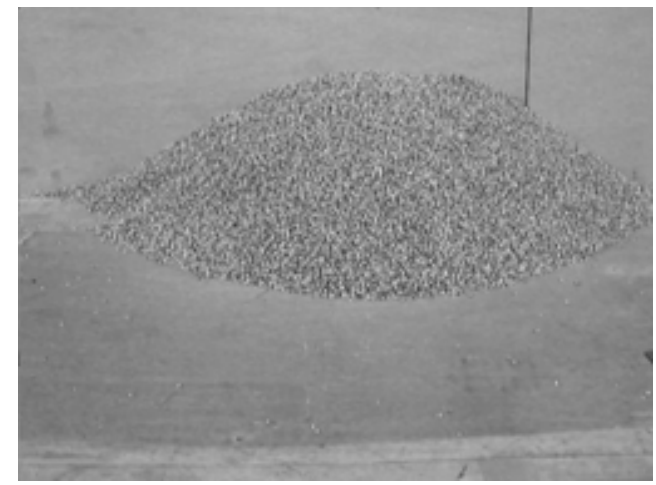

Fig.14 Experimental pile

Fig.15 shows the first scooping and V shape path to the loading position. Gray bold line represents the edge of the pile and the loading position is (-500 1000) and angle $\theta_{e}=70$.

As the candidate of the next scooping position, two positions are considered. One is $P+: 200 \mathrm{~mm}$ left of the first scooping position and the other is $P-: 200 \mathrm{~mm}$ right. Because the width of the bucket of YZ-2 is $250 \mathrm{~mm}$, these next positions are overlapped to the first scooping area. Fig.16 shows the estimate moment $M$ on the bucket. Dark triangle and grey square represent $M$ for $P+$ and $P$-respectively. $M$ at $P+$ becomes 0 at $\theta_{\mathrm{s}}=-15.8$ and $M$ at P- becomes 0 at $\theta_{\mathrm{s}}=25.8$. These scooping angles seem to be perpendicular to the edge of the pile.

Fig.17 shows the V shape path for candidate of the next scooping positions $P+$ and $P$-. Bold line and thin line represent the $\mathrm{V}$ shape path for $P+$ and $P$ - respectively. The total length of the path is $2950 \mathrm{~mm}$ for $P+$ and $5075 \mathrm{~mm}$ for $P$-. In this case, $P+$ has great advantage in the travel length of the loader. It is the additive effect of the distance and the direction between the scooping position and the loading position. $P+$ should be selected as the next scooping point in this case.

Fig.18 shows V shape path in same situation in Fig.@ except loading position and angle. In this case, $P_{e}$ is set at (-500 500) and $\theta_{e}=70$. Because the position $P+$ is too close to the loading position, the path from $P+$ to the loading position cannot fill the requirement on $l_{3}$ to be positive. Because $P+$ does not fill the requirement, $P$ - has to be selected as the next scooping point in this case.

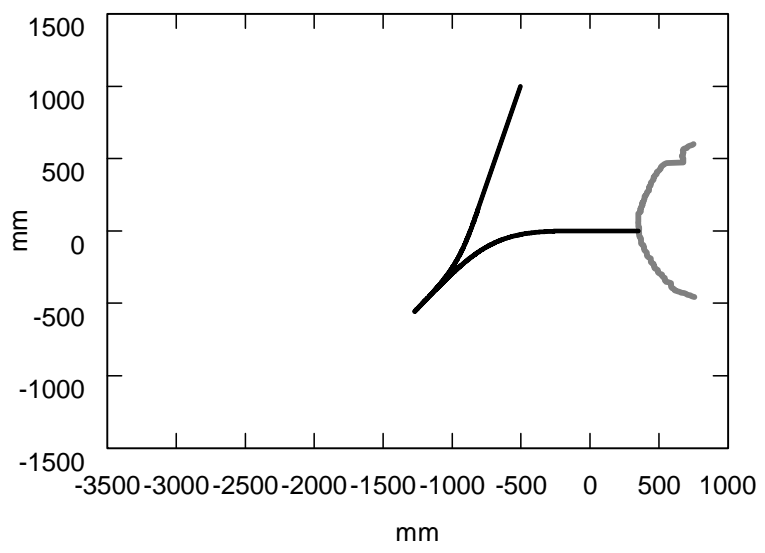

Fig. 15 The First Scooping and V shape Path

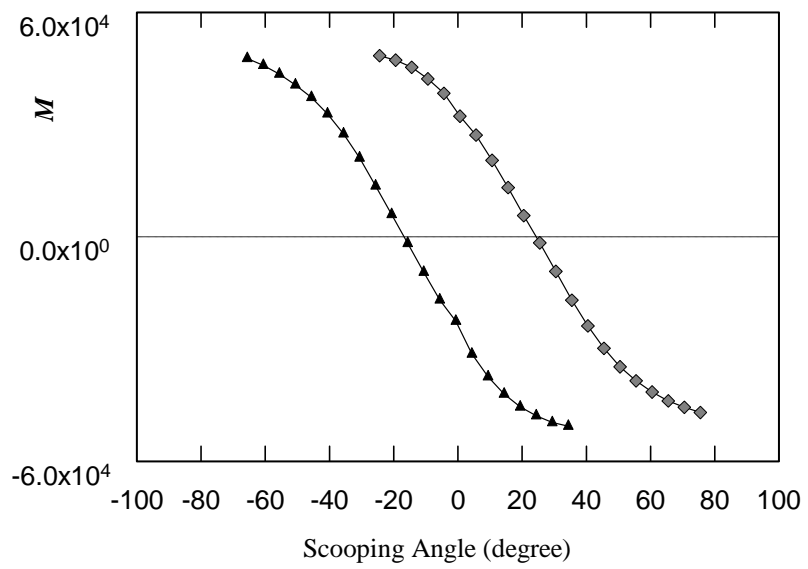

Fig. 16 Moment on the bucket 


\section{CONCLUSION}

An itemized outline of the proposed method follows.

(1) The scooping direction is determined based on the resistance force applied on the bucket during scooping motion. The irregular shape of the pile is expressed numerically by a column model. The unbalance of the resistance force applied on the bucket is estimated using the column model and bucket trajectory. The result of this estimation is used to determine the appropriate scooping direction.

(2) The planned path consists of two symmetrical clothoid curves and three line segments. To simplify the generation of the clothoid, the curvature is limited to several values. The lengths of line segments are determined through optimization.

(3) The total length of the V shape is affected by the angle at the switch back point. The angle providing the minimum length of the path is obtained by comparison among these at different angle of the switch back point.

(4) V shape path by the proposed method is affected from the distance and direction between the scooping point and the loading point. Appropriateness of the planned path depends on the length of the path as well as the practicability on of reachable.

\section{REFERENCES}

[1] Sarata, S.: Model-based Task Planning for Loading Operation in Mining. Proc. of IROS, pp439-445, 2001

[2] Sarata, S.: Research and Development on Unmanned Loading Operation by Wheel Loader, Proc. Rapid Mine Development, pp249-258, 2001

[3] Gocho,T. et al: Autonomous Wheel-Loader in Asphalt Plant, Proc. 9th ISRAC, 1992

[4] Oshima, H. et al :Automation of Loading and Hauling Work in Mining and Quarry, Komatsu technical report, Vol.43, No.1, pp.27-39, 1997 (in Japanese)

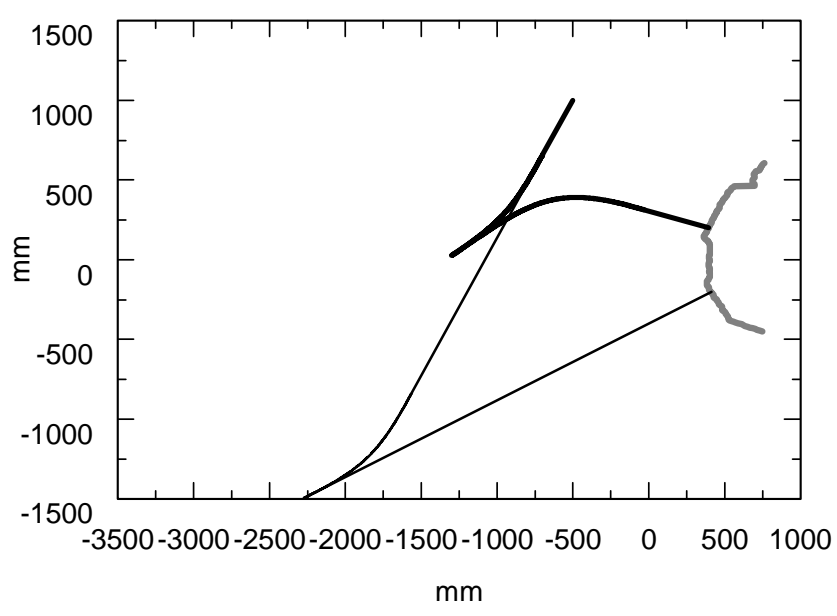

$P_{e}(-5001000), \theta_{e}=70$

Fig.17 V shape path for $P+$ and $P$ -

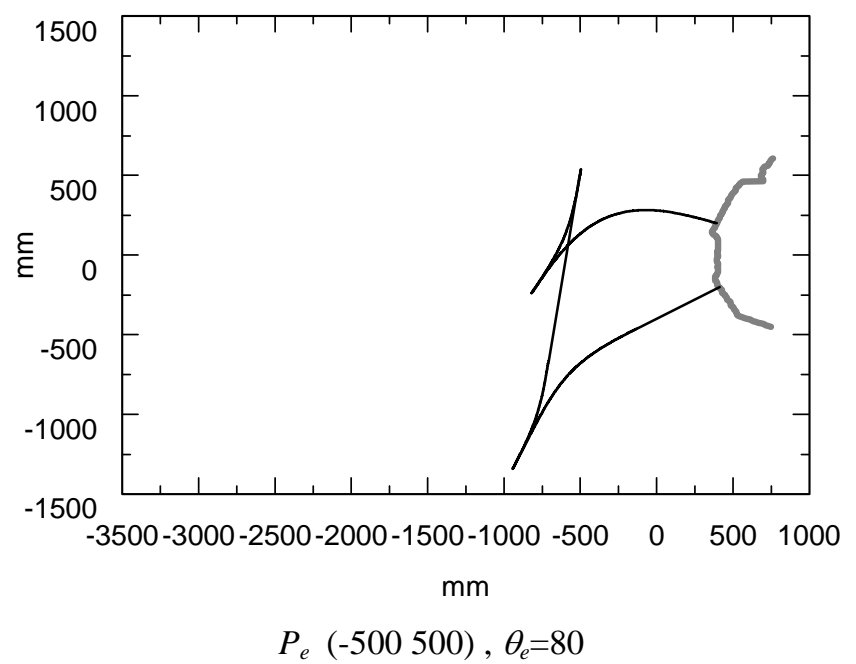

Fig.18 V shape path for $P+$ and $P$ - 